\title{
Characterization of Self-Assembled CdTe/ZnTe Quantum Dots
}

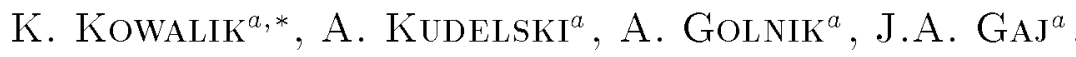 \\ G. KARCZEWSKI ${ }^{b}$ AND J. KOSSUT ${ }^{b}$ \\ ${ }^{a}$ Institute of Experimental Physics, Warsaw University \\ Hoża 69, 00-681 Warszawa, Poland \\ ${ }^{b}$ Institute of Physics, Polish Academy of Sciences \\ al. Lotników 32/46, 02-668 Warszawa, Poland
}

\begin{abstract}
We present microluminescence investigations of self-assembled $\mathrm{CdTe} / \mathrm{ZnTe}$ quantum dots. The dots proprieties resulting from our studies are: values of optical in-plane anisotropy parameters (electron - heavy hole exchange splitting and orientation of anisotropy) and value of effective Lande factor. Parameters giving information about in-plane anisotropy possess random distribution of values with the exchange splitting from 0 to $240 \mu \mathrm{eV}$. The effective Lande factor values for our dots are around $g^{*}=-3.2$ with a scatter of about $18 \%$. Some PL lines exhibit sudden jumps of energetic position, related to variation of the charge state in their neighborhood.
\end{abstract}

PACS numbers: 78.67.Hc, 71.70.Ej

\section{Introduction}

Quickly developing field of quantum cryptography, as well as perspectives of quantum computing, created a demand for sources of entangled photon pairs. Semiconductor quantum dots as potential sources of such pairs have the advantage of easy incorporation in electronic or optoelectronic integrated circuits. Experimental efforts undertaken mainly for III-V quantum dot systems, resulted so far in obtaining photon pairs with correlated polarization but no entanglement. These difficulties can be caused by anisotropy of individual quantum dots. In this work we present the results of polarization resolved studies of luminescence lines originated from single $\mathrm{CdTe} / \mathrm{ZnTe}$ quantum dots. Luminescence was measured also as a function of magnetic field, temperature, and time.

*corresponding author; e-mail: kkowalik@fuw.edu.pl 


\section{Sample and experiment}

The investigated sample was grown by molecular beam epitaxy [1] in the Institute of Physics of Polish Academy of Sciences in Warsaw. A $1 \mu \mathrm{m} \mathrm{ZdTe}$ buffer was deposited on (001) oriented GaAs substrate. The CdTe self-assembled quantum dots were produced by ALE deposition of two monolayers of CdTe on the buffer. The structure was completed by deposition of a $0.05 \mu \mathrm{m}$ ZnTe cap (inset in Fig. 1).

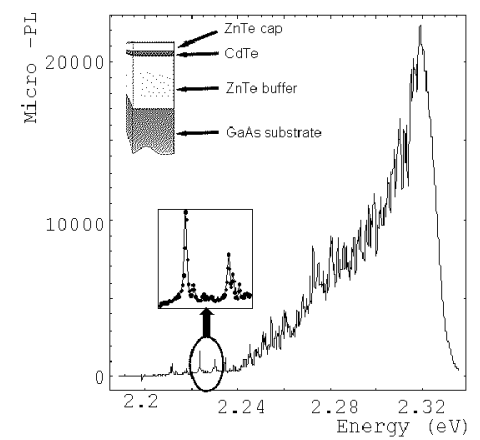

Fig. 1. Microluminescence spectrum (full energy range) with sample structure and a zoom of lines chosen for analysis.

We used a microluminescence setup similar to that presented by Kudelski et al. [2]. The sample was mounted on a flat sector of a small-sized mirror objective [3] and both were placed inside the cryostat of the superconducting magnet enabling us to perform measurements of luminescence in Faraday configuration. The used microscope has magnification of 100 and numerical aperture 0.7 . The spatial resolution was of the order of $1 \mu \mathrm{m}$. An overall photoluminescence spectrum of the sample is shown in Fig. 1. For studies of individual dot lines, we selected suitable fragments of the spectrum, where the lines were not too close to each other. Mechanical stability of the experimental setup and the properties of the microobjective gave the possibility to follow the luminescence of individual quantum dots during many hours of measurements. An argon laser line (488 nm) was used for excitation. A glass plate sent the beam into the microscope at a small angle to the optical axis. A circular analyzer placed in front of the spectrometer was used in Zeeman effect measurements. For anisotropy measurements an additional linear polarizer was placed in the luminescence beam.

\section{Theoretical outline}

Optical anisotropy of quantum dots is usually described in terms of electronhole exchange interaction, using following Hamiltonian [4]: 


$$
H_{\text {exchange }}=-\sum_{i=x, y, z}\left(a_{i} J_{\mathrm{h}, i} S_{\mathrm{e}, i}+b_{i} J_{\mathrm{h}, i}^{3} S_{e, i}\right),
$$

where $a_{i}, b_{i}$ are parameters and $J_{\mathrm{h}, i}, S_{\mathrm{e}, i}$ are matrices of angular momentum for heavy-hole and electron, respectively. In excitonic base $| \pm 1\rangle,| \pm 2\rangle$ it can be written as:

$$
H_{\text {exchange }}=\frac{1}{2}\left(\begin{array}{cccc}
\delta_{0} & \delta_{1} & 0 & 0 \\
\delta_{1} & \delta_{0} & 0 & 0 \\
0 & 0 & -\delta_{0} & \delta_{2} \\
0 & 0 & \delta_{2} & -\delta_{0}
\end{array}\right),
$$

where all parameters (related with $a_{i}, b_{i}$ from the previous formula) determine directly excitonic splittings. From optical measurements of luminescence, involving only the bright exciton, nothing but $\delta_{1}$ can be obtained. Parameter $\delta_{1}$ is the value of the exchange splitting of the two bright exciton states. Because of the relation: $\delta_{1}=0.75\left(b_{x}-b_{y}\right)$, this term does not vanish in case, when perpendicular axes in the sample plane are not equivalent. Two bright exciton states obtained from the diagonalization of the Hamiltonian, possess linear polarizations perpendicular to each other.

\section{Results}

For the sample characterization three sorts of experiments were done. Polarization resolved measurements were made to determine the value of the exchange splitting, from Zeeman effect studies the value of effective Lande factor was calculated. We also investigated temporal evolution of the spectra to distinguish the origin of the lines.
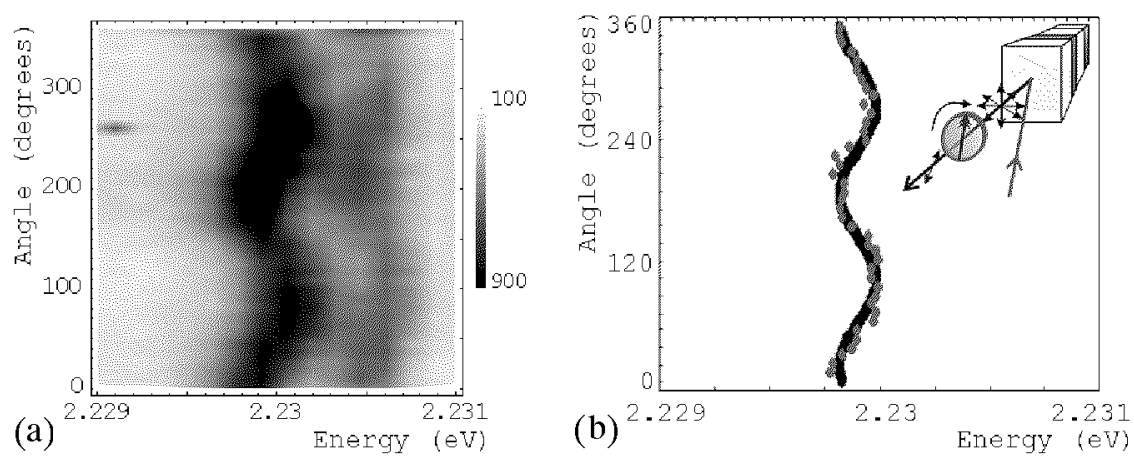

Fig. 2. Map of variation of spectra with polarization angle (a). Line position versus polarization angle fitted with cosine function (in right corner - idea of anisotropy measurements) (b).

We measured the anisotropy of QDs as a variation of the spectra with the polarization angle. The result of such an experiment is shown in Fig. 2a. From theoretical consideration we expected to have two lines split by exchange interaction, 
but because of small value of the splitting compared to lines widths, only one line was observed. Fortunately we know additionally that these two components of the line should possess opposite polarizations. Therefore in polarization resolved studies the exchange interaction manifests itself in periodic movements of this single line position. For one chosen line it is clearly seen in Fig. 2a. From the fitting of the line position vs. polarization angle (see Fig. 2b) two parameters of anisotropy can be obtained. The first - a distance between minimum and maximum of fitted cosine equal to the value of exchange splitting. The second - the phase of the cosine which describes orientation of the anisotropy. Comparing results obtained for several QDs we observed random distribution of both anisotropy parameters (Fig. 3). The orientation of the anisotropy change from dot to dot in the whole range and the exchange splitting changes from zero to $240 \mu \mathrm{eV}$.

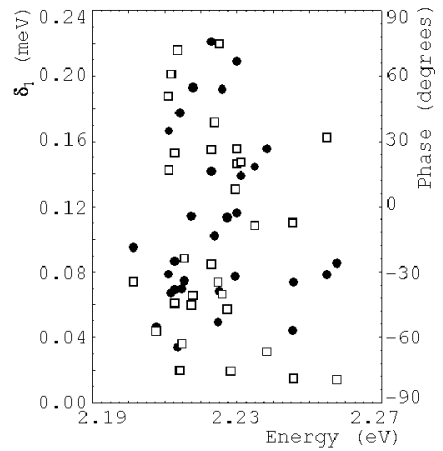

Fig. 3. Anisotropy parameters for investigated dots: values of exchange splitting $\delta_{1}$ (circles) and of orientation of anisotropy $\varphi$ (squares) vs. energy positions of lines.

Photoluminescence measurements in magnetic field gave us the possibility to obtain values of Zeeman splitting for individual quantum dots. A map showing variation of the PL spectrum of a chosen line is shown in Fig. 4a. We obtained effective Lande factor $g^{*}$ values from fits of the line position as a function of magnetic field (Fig. 4b). The determined values vary around $g^{*}=-3.2$ with a scatter of about $18 \%$. These values are systematically greater than those reported by Besombes et al. [5] for a CdTe/ZnTe self-assembled QD system grown in Stransky-Krastanov mode.

Some individual dot lines in self-assembled QD system are known to experience sudden jumps of their energy position. This effect, first observed for the CdTe/ZnTe system by Besombes et al. [6], is usually explained by changes in the charge state of some centers lying close to the observed dot. We observed such shifts of the PL lines in our experiments as shown in Fig. 5. The analysis of such jumps may help in identifying lines coming from the same quantum dots since they are expected to shift synchronously. 

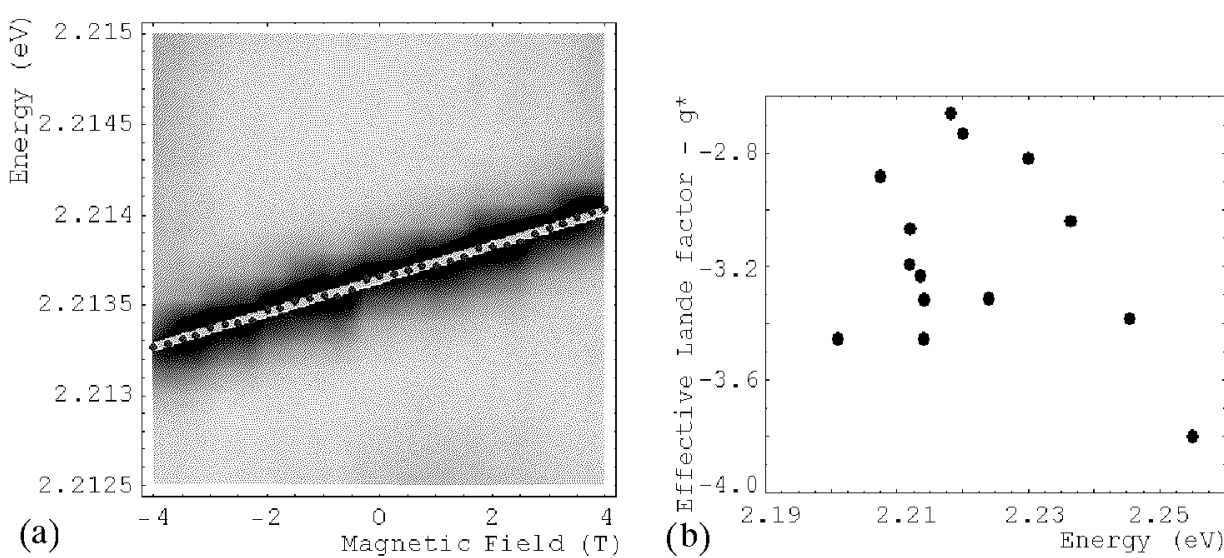

Fig. 4. Zeeman effect map for chosen line: points - fitted line position, solid line Zeeman effect fit (a). Values of effective Lande factor vs. line energy (b).

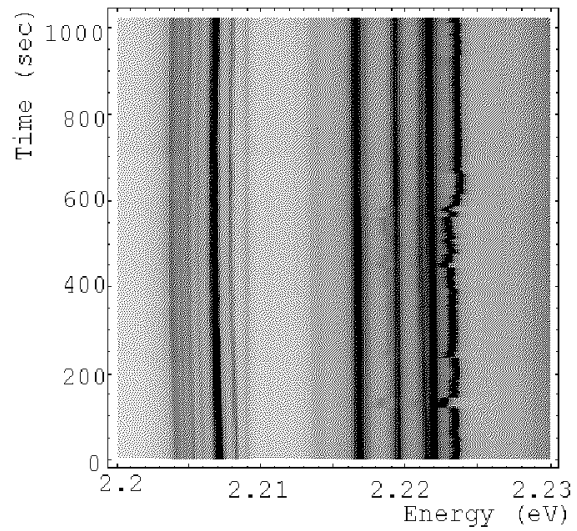

Fig. 5. Temporal evolution of spectrum.

\section{Conclusions}

A characterization study of a CdTe/ZnTe self-assembled quantum dot system was performed. Anisotropic exchange splittings up to $240 \mu \mathrm{eV}$ were measured for individual quantum dot lines, as well as Zeeman splittings in Faraday configuration. Effective $g$-factor values around $g^{*}=-3.2$ with a scatter of about $18 \%$ were determined. No correlation between the determined parameter values was found. Some PL lines exhibit sudden jumps of energetic position.

\section{Acknowledgment}

This work was supported by the State Committee for Scientific Research grant PBZ-KBN-044/P03/2001. 


\section{References}

[1] G. Karczewski, S. Maćkowski, M. Kutrowski, T. Wojtowicz, J. Kossut, Appl. Phys. Lett. 74, 3011 (1999).

[2] A. Kudelski, A. Golnik, J.A. Gaj, S. Maćkowski, G. Karczewski, J. Kossut, in: Proc. 25th Internat. Conf. Phys. Semicond., Osaka 2000, Eds. N. Miura, T. Ando, Springer Proc. in Physics, 87, Springer Verlag, Berlin 2001, p. 1249.

[3] J. Jasny, J. Sepiol, T. Irngartinger, M. Traber, A. Renn, U.P. Wild, Rev. Sci. Instrum. 67, 1425 (1996).

[4] H.W. van Kasteren, E.C. Cosman, W.A.J.A. van der Poel, C.T. Foxon, Phys. Rev. $B$ 41, 5283 (1990).

[5] L. Besombes, L. Marsal, K. Kheng, T. Charvolin, Le Si Dang, A. Wasiela, H. Mariette, J. Cryst. Growth 214/215, 742 (2000).

[6] L. Besombes, K. Kheng, L. Marsal, H. Mariette, Phys. Rev. B 65, 121314(R) (2002). 